\title{
A New Clutter Rejection Algorithm for Doppler Ultrasound
}

\author{
Guy Cloutier*, Member, IEEE, Danmin Chen, and Louis-Gilles Durand, Senior Member, IEEE
}

\begin{abstract}
Several strategies, known as clutter or wall Doppler filtering, were proposed to remove the strong echoes produced by stationary or slow moving tissue structures from the Doppler blood flow signal. In this study, the matching pursuit (MP) method is proposed to remove clutter components. The MP method decomposes the Doppler signal into wavelet atoms that are selected in a decreasing energy order. Thus, the high-energy clutter components are extracted first. In the present study, the pulsatile Doppler signal $s(n)$ was simulated by a sum of random-phase sinusoids. Two types of high-amplitude clutter signals were then superimposed on $s(n)$ : time-varying low-frequency components, covering systole and early diastole, and short transient clutter signals, distributed within the whole cardiac cycle. The Doppler signals were modeled with the MP method and the most dominant atoms were subtracted from the time-domain signal $s(n)$ until the signal-toclutter $(S / C)$ ratio reached a maximum. For the low-frequency clutter signal, the improvement in $S / C$ ratio was $19.0 \pm 0.6 \mathrm{~dB}$, and $72.0 \pm 4.5$ atoms were required to reach this performance. For the transient clutter signal, ten atoms were required and the maximum improvement in $S / C$ ratio was $5.5 \pm 0.5 \mathrm{~dB}$. The performance of the MP method was also tested on real data recorded over the common carotid artery of a normal subject. Removing 15 atoms significantly improved the appearance of the Doppler sonogram contaminated with low-frequency clutter. Many more atoms (over 200) were required to remove transient clutter components. These results suggest the possibility of using this signal processing approach to implement clutter rejection filters on ultrasound commercial instruments.
\end{abstract}

Index Terms-Clutter filter, echocardiography, matching pursuit method, simulation, spectral method, time-frequency representation technique, ultrasonography, wall filter, wavelets.

\section{INTRODUCTION}

A $S$ RECENTLY reviewed [1], several strategies were proposed in the field of medical ultrasound to remove the strong echoes produced by stationary or slow moving structures from the blood flow signal. The strategies applied to the Doppler signals are known as clutter or wall filters.

Manuscript received June 12, 2002; revised November 15, 2002. This work was supported by a research scholarship from the Fonds de la Recherche en Santé du Québec, and by the Natural Sciences and Engineering Research Council of Canada under Grant 138570. The Associate Editor responsible for coordinating the review of this paper and recommending its publication was M. Insana. Asterisk indicates corresponding author.

*G. Cloutier is with the Laboratory of Biorheology and Medical Ultrasonics, Research Center, Centre hospitalier de l'Université de Montréal, 2099 Alexandre de Sève, Room Y-1619, Montréal, QC H2L 2W5, Canada, and also with the Department of Radiology, University of Montreal, Montréal, QC H3C 3J7, Canada (e-mail: guy.cloutier@umontreal.ca).

D. Chen and L.-G. Durand are with the Laboratory of Biomedical Engineering, Clinical Research Institute of Montreal, QC H2L 2W5, Canada.

Digital Object Identifier 10.1109/TMI.2003.809059
Currently, the methods used by ultrasound manufacturers are mainly based on high-pass finite and infinite impulse response filters, and regression filters. These methods are applied to process either spectral Doppler [continuous-wave (CW) and pulsed-wave (PW)] or color Doppler (velocity mapping and power angiography) flow data. In the context of color flow [2], [3], these methods have limitations that are mainly related to the short duration of the signal available for data processing (the number of samples available, typically six to 12 , limits the number of coefficients, or filter order, and thus the sharpness of the transition from the passband to the stopband). For spectral Doppler, the signal duration is less a concern but as described below, new applications motivate the need for novel signal processing strategies.

For most clinical scans, the cutoff frequency of the high-pass filter is usually selected by the operator according to the axial flow characteristics and importance of the clutter signal. Clutter components detectable on spectral Doppler or sonogram (timefrequency display of Doppler signals usually computed with the fast Fourier algorithm) are produced either by static interfaces, vessel wall pulsatility, muscular tissue movements, or displacement of heart valves when cardiac applications are considered. The removal of clutter on CW Doppler sonogram is more critical than in PW mode or color flow because the former method registers echoes all along the ultrasound beam. In PW and color modes, the Doppler sample volume(s) is (are) usually positioned in the flow stream and clutter results from echoes detected mainly by the sidelobes of the ultrasound beam.

In theory, for static interfaces, no clutter should be detected because the sonogram only displays reflectors in movement that produce a Doppler frequency shift. However, in practice, this may not be the case if the static interfaces producing echoes at $0 \mathrm{~Hz}$ are not properly removed. For instance, the static clutter may be detectable on the sonogram because the sidelobes of the spectral window used to process the Doppler time-domain signal artificially produce frequency harmonics. For a typical Hamming window, the clutter signal is detected on the sonogram when it exceeds the noise floor by at least $40 \mathrm{~dB}$ (the sidelobes for that window are $-40 \mathrm{~dB}$ below the magnitude of the main lobe centered at $0 \mathrm{~Hz}$ ) [4]. For moving interfaces such as vessel walls, muscular tissue, and heart valves, the clutter frequency shift is a function of the vibration velocity. In this case, the clutter components are nonstationary and they are produced by both the main and sidelobes of the window function. It may be mentioned that trembling of the probe hold by the clinician may also produce clutter. For simplicity, only moving reflectors 
are considered in the present study. Moreover, the study is limited to spectral Doppler clutter removal (although the method may be applicable for color Doppler).

As recently emphasized with clinical examples [5], clutter vibrations can still impair the diagnostic accuracy of Doppler ultrasound, even if modern instruments are used. A solution may be the use of ultrasound contrast agent combined with harmonic imaging. This modality was shown to improve the signal-to-clutter $(S / C)$ ratio of spectral Doppler, color Doppler, and power Doppler angiography. Nevertheless, in new developments, such as vector Doppler that requires the estimate of flow from different angles [6], there is a need for new clutter rejection filter designs. For instance, the flow components close to perpendicular with respect to the ultrasound beam produce low-frequency shifts that may overlap the frequency range covered by the clutter filter. Because the Doppler shift from different angles is required to reconstruct the two-dimensional (2-D) or three-dimensional (3-D) flow patterns, this adds to the complexity of the signal processing that is required to optimize the $S / C$ ratio. For instance, the use of classical high-pass clutter filters can result in the removal of relevant flow information that is required to reconstruct the velocity vectors. Such a situation would occur because, to our knowledge, classical filters are not adaptable to the time-varying characteristics of the Doppler flow signals (the filter cutoff frequency remains constant all over the flow cycle). In addition to the need for vector Doppler, the detection of vibrating muscles with PW Doppler instruments is another field of application where new clutter filtering strategies may be required [7]. A method based on the modeling of the wall-motion signal (autoregressive linear prediction, model order $=1$ ) and subsequent subtraction from the unfiltered PW Doppler components was recently proposed [8].

In the present study, the matching pursuit (MP) method is introduced to remove clutter components while preserving, when possible, the integrity of the low-velocity flow signal. As described below, the MP method decomposes the signal into basic wavelets having a finite time duration and frequency span. Consequently, by selectively removing relevant atoms due to clutter, low-amplitude and low-frequency flow signals not superimposed over the clutter vibration should remain intact after applying the MP method. The feasibility of the technique is demonstrated with simulated and real PW Doppler data.

\section{METHOD}

\section{A. Simulation of Doppler Signals With Clutter Artifacts}

The Doppler signal $s(n)$, sampled at $9.5 \mathrm{kHz}$, was simulated by a sum of sinusoids [9], whose frequencies $f_{m}$ and $f_{p}$ subdivide the frequency range $\left[0, f_{m \max }(n)\right]$ or $\left[0, f_{p \max }(n)\right]$ in $M+P$ bins of equal width $\Delta f=1 \mathrm{~Hz}$ [see below the definitions of $f_{m \max }(n)$ and $\left.f_{p \max }(n)\right]$. The duration of the simulated flow cycle was $857 \mathrm{~ms}$ (8142 samples). As observed experimentally during clinical scans, two types of high-amplitude clutter components were superimposed on $s(n)$, a time-varying low-frequency signal and short clutter transients, which produce in the frequency domain wide spectral lines. The Doppler signal of one cardiac cycle was modeled as

$$
\begin{aligned}
s(n)= & \sum_{m=1}^{M} \gamma_{m}(n-327) \cos \left[2 \pi f_{m}(n-327)+\phi_{m}\right] \\
& \text { for } 327 \leq n<3585 \text { (zero otherwise) } \\
& +\gamma_{0} \sum_{p=1}^{P} \exp \left(\frac{-f_{p}^{2}}{2 \times B W(n)^{2}}\right) \cos \left[2 \pi f_{p} n+\psi_{p}\right] \\
& \text { for } 0 \leq n<3911(\text { zero otherwise }) \\
& +\sum_{q=1}^{Q} \sqrt{E\left(n_{q}\right)} \delta\left(n-n_{q}\right), \text { for } 0 \leq n<8142 .
\end{aligned}
$$

Equation (1a) describes the clutter-free Doppler signal, where $M$ is the number of sinusoids between $\left[0, f_{m \max }(n)\right], \gamma_{m}(r=$ $n-327)=\sqrt{2 S(r, k) \Delta f y_{m}}$ is a Rayleigh distributed random variable, and $f_{m}=(m-0.5) \Delta f$. The random phase $\phi_{m}$ is uniformly distributed between $[0,2 \pi] ; S(r, k)$, which is defined below, is the time-varying theoretical power spectral density function of the clutter-free signal; $\Delta f=f_{m \max }(n) / M$ is an incremental frequency domain step; and $y_{m}$ is a $\chi$-squared random variable with two degrees of freedom. In the present study, the maximum value of $M$ at peak systole $=4500$ and it was chosen to respect the criterion defined by Mo and Cobbold [9]. Based on this criterion, the simulated Doppler signals were assumed to be Gaussian distributed in amplitude.

The second term in (1b) was used to simulate the time-varying low-frequency clutter components. In this equation, $P$ is the number of sinusoids between $\left[0, f_{p \max }(n)\right]$; $\gamma_{0}$ is a scaling amplitude defined by the $S / C$ energy ratio selected; $f_{p}=(p-$ $0.5) \Delta f$, where $\Delta f=f_{p \max }(n) / P ; B W(n)$ is the bandwidth of the clutter component that varies as a function of the timing within the cardiac cycle $\left(B W(n)=2 \sqrt{f_{p \max }(n) / \pi}\right.$; and $\psi_{p}$ is the uniformly distributed random phase between $[0,2 \pi]$. The maximum value of $P$, in early systole and late diastole, was 300. Equation (1c) defines the short transient clutter components, which were modeled as a Poisson point process of successive Dirac impulses. The parameter $Q$ is the number of samples of the signal $s(n), E\left(n_{q}\right)$ is the impulse energy of the clutter components, and the $\left[n_{q}\right]$ are assumed randomly distributed with a fixed appearance rate $R$. The parameter $\sqrt{E\left(n_{q}\right)}$ was selected as the absolute value of the maximum of $\gamma_{m}(n)$, for $1 \leq m<M$ and $0 \leq n<8142$.

In the definition of the parameter $\gamma_{m}(n)$ given above, the theoretical power spectral density of the clutter-free Doppler signal, as a function of time, is given by [9]

$$
\begin{gathered}
S(r, k)=A(r)\left[f_{m \max }(r)-f_{k}\right]^{2} \exp \left(-B(r)\left[f_{m \max }(r)-f_{k}\right]^{2}\right) \\
0 \leq r<3258 \text { and } 0 \leq k<f_{m \max }(r)
\end{gathered}
$$

where $f_{m \max }, A$, and $B$ are time-varying parameters representing the maximum frequency of the sinusoids $f_{m}$, the power scaling factor, and the bandwidth factor, respectively. The parameter $f_{k}$ is the frequency corresponding to the spectral index $k$. In (2), the greater is the value of $B$, the narrower is the spectrum. The total power of the time-varying spectra $S(r, k)$ were normalized to unity by varying the value of $A$. The functions 


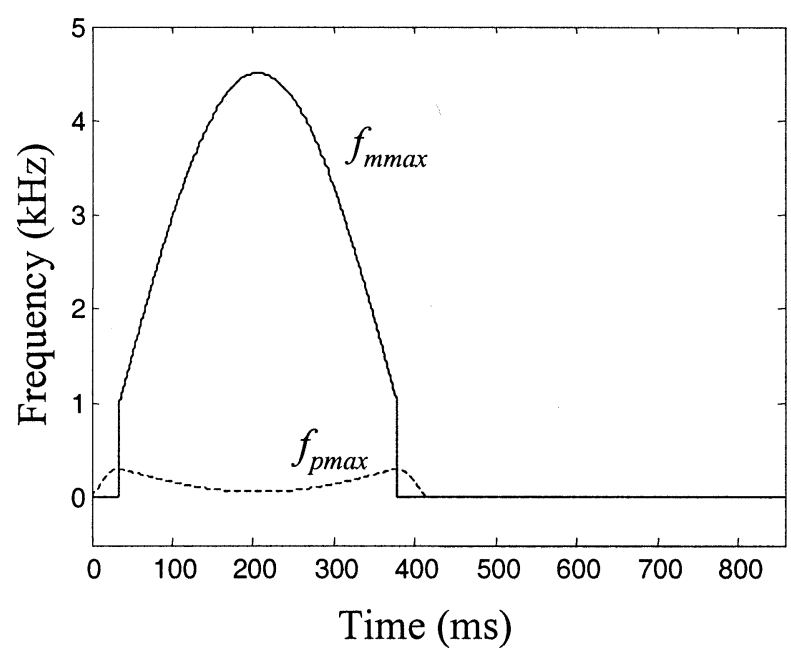

Fig. 1. Basic time-frequency functions $f_{m \max }(n)$ (full line) and $f_{p \max }(n)$ (dotted line) that were used to generate the simulated Doppler signal $s(n)$.

$A(r)$ and $B(r)$ in (2) were set to values obtained by the procedure described by Guo et al. [10].

Fig. 1 shows the time-frequency functions $f_{m \max }(n)$ and $f_{p \max }(n)$ that were used to generate the Doppler signal $s(n)$ given by (1). The function $f_{m \text { max }}$, defined between 34 and $377 \mathrm{~ms}$, was computed by

$f_{m \max }(r=n-327)=3.5 \sin \left(\frac{2 \pi f_{1} r}{F}\right)+1$,

$$
\text { for } 0 \leq r<3258 \text { (zero otherwise) }
$$

where $f_{1}=1.46 \mathrm{~Hz}, F=9500$ is the sampling frequency in hertz, and $f_{m \text { max }}$ is in kilohertz. The maximum frequency waveform $f_{p \max }$, defined between 0 and $411 \mathrm{~ms}$, describes the contour of the low-frequency clutter components. For $f_{p \text { max }}$ in kilohertz and $f_{2}=7.3 \mathrm{~Hz}$, it was calculated by

$$
\begin{aligned}
f_{p \max }(n)= & 0.3 \cos \left(\frac{2 \pi f_{2} n}{F}+\frac{3}{2} \pi\right) \\
& \text { for } 0 \leq n<327 \\
= & 0.3-0.25 \sin \left(\frac{2 \pi f_{1}(n-327)}{F}\right) \\
& \text { for } 327 \leq n<3585 \\
= & 0.3 \cos \left(\frac{2 \pi f_{2}(n-3585)}{F}\right) \\
& \text { for } 3585 \leq n<3911 \text { (zero otherwise) } .
\end{aligned}
$$

When present, a total of ten transient clutter components was simulated within the cardiac cycle. Depending on the simulated conditions (Doppler signals with low-frequency clutter or Doppler signals with transient clutter), $\gamma_{0}$ and $E\left(n_{q}\right)$ in (1) could be either of finite values or zero values. For each simulated condition, a total of five independent cardiac cycles (the random parameters were reinitiated for each cycle) was generated for averaging purpose. No background noise was added to the simulated signals described by (1).

\section{B. MP Method}

The MP method [11] is based on a dictionary that contains a family of Gabor wavelet functions called time-frequency atoms.

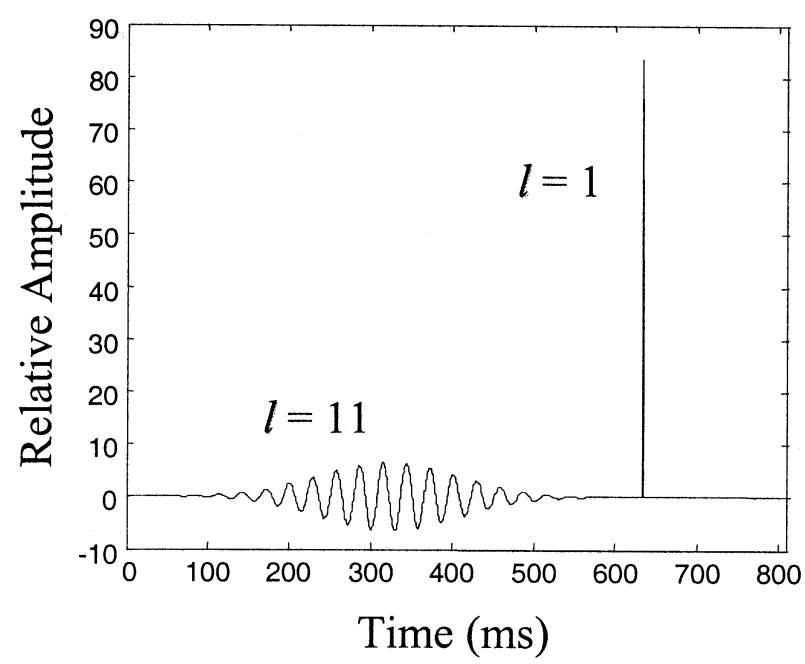

Fig. 2. Example of two time-domain atoms: one with $l=11$ [ $l$ is the octave of the scale $s_{i}$ in (8)], at time position $323 \mathrm{~ms}$, and another with $l=1$, at time position $634 \mathrm{~ms}$.

The decomposition of a signal is performed by projecting it over the function dictionary iteratively, and by selecting the atoms or wavelets that can best match the local waveform of the signal. The MP method represents a discrete signal $s(n)$ with $N$ samples as

$$
\begin{aligned}
s(n) & =\sum_{i=1}^{+\infty} \alpha_{i} h_{i}(n) \\
\text { with } h_{i}(n) & =\beta_{i} g_{i}(n) u_{i}(n) \\
g_{i}(n) & =g\left(\frac{n-p_{i}}{s_{i}}\right) \\
\text { and } u_{i}(n) & =\cos \left(\frac{2 \pi f_{i} n}{N}+\theta_{i}\right) .
\end{aligned}
$$

In the above equations, $\alpha_{i}$ are the expansion coefficients that represent, when squared, the part of the signal energy associated with the atom $h_{i}(n)$. In (6), the atoms $h_{i}(n)$ are given by the product of the wavelet envelope function $g_{i}(n)$ with the sinusoids $u_{i}(n)$. The scale factors $s_{i}$ are used to control the width of the envelope of $h_{i}(n)$, and $p_{i}$ give the temporal position of each atom. To reduce the computational burden, the scale was limited to $s_{i}=2^{l}$, where $l$ is the octave of the scale $s_{i}$, which varies between 0 and $\log _{2} N$. Hence, only the scales $s_{i}$ that satisfy this relation and lie in $[1, N]$ were selected from the dictionary. It may be of interest to note that the Gabor dictionary provides a finer time-frequency resolution than a dictionary based on orthogonal wavepacket functions because the Gabor atoms, which are characterized by a Gaussian shape, can have either a good time resolution (and poor frequency resolution), a good frequency resolution (and poor time resolution), or a compromise between both (2-D Gaussian spots). As illustrated in Fig. 2, a low value of $l$ corresponds to short transient atoms having a wide frequency bandwidth, whereas a high value of $l$ adequately represents long duration signals with a narrow frequency bandwidth. In (6), the parameters $\beta_{i}$ are normalizing factors to keep the norm of $h_{i}(n)$ equal to 1 . The parameters $f_{i}$ and $\theta_{i}$ in (8) are the discrete fre- 
quency and phase of the cosine discrete function $u_{i}(n)$, respectively. In our application

$$
g_{i}(n)=2^{\frac{l}{4}} e^{-\pi\left(\frac{n-p_{i}}{s_{i}}\right)^{2}}
$$

is the Gabor function [11].

An important property of the MP method is the fact that it finds the time-frequency atoms in a decreasing energy order. Thus, the higher-energy components of the signal due to the clutter are extracted first. Once an atom was found, it was subtracted from the simulated time-domain signal $s(n)$ to get a new signal $s_{d}(n)$ (decontaminated signal). After having selected a given number of atoms to optimize the signal-to-clutter $(S / C)$ ratio, the Doppler sonogram of $s_{d}(n)$ was computed to display the simulated signal. The sonogram was evaluated by using the Welch method [12]. The fast Fourier transform (FFT) was applied on sections of $10 \mathrm{~ms}$ obtained after windowing the signal $s_{d}(n)$ with the Hamming function. A lag of $0.5 \mathrm{~ms}$ was used between successive FFTs and zero padding to 1024 samples was performed.

\section{Computation of the Performance of the MP Method in Terms of S/C Ratio}

Let us consider the simulated signal $s(n)$ in (1) to be the sum of two components: $s_{1}(n)$ and $s_{2}(n)$, where $s_{1}(n)$ is the clutter-free signal [(1a)], and $s_{2}(n)$ is the clutter components [either the low-frequency signal of (1b) or the transient component of (1c)]. In the current study, no simulation was performed with both low-frequency and transient clutter components. By iteratively applying the MP method to extract atoms from $s(n)$, one could obtain the decontaminated signal $s_{d}(n)$ by computing

$$
s_{d}(n)=\sum_{i=1}^{L} s(n)-\alpha_{i} h_{i}(n), \text { for } 0 \leq n<N
$$

where $L$ is the number of atoms selected, $\alpha_{i}$ are the expansion coefficients, $h_{i}(n)$ is the atom extracted at the iteration $i$, and $N=8142$ is the number of samples of the signal. After removing a given number of atoms $L$, the $S / C$ ratio in decibels was calculated as follows:

$$
\begin{aligned}
S / C \text { ratio } & =10 \times \log _{10}\left(\frac{\sum_{n=0}^{N} s_{1}(n)^{2}}{\sum_{n=0}^{N}\left(s_{d}(n)-s_{1}(n)\right)^{2}}\right) \\
& =10 \times \log _{10}\left(\frac{\sum_{n=0}^{N} s_{1}(n)^{2}}{\sum_{n=0}^{N}\left[s_{2}(n)-\sum_{i=1}^{L} \alpha_{i} h_{i}(n)\right]^{2}}\right) .
\end{aligned}
$$

In the current study, the $S / C$ ratios before removing atoms (i.e., $i=0$ ) were $-17.3 \pm 0.6 \mathrm{~dB}$ for the low-frequency high amplitude clutter and $10.5 \pm 0.4 \mathrm{~dB}$ for the transient clutter. These values were computed over five simulated cardiac cycles. Although the $S / C$ ratio of low-frequency wall movements can reach -40 to $-60 \mathrm{~dB}$ at frequencies below $10 \mathrm{MHz}$ [1], the scattered power of the tissue clutter component was selected smaller here to test the robustness of the MP algorithm for clutter amplitude approaching the flow signal amplitude. For instance, as presented in Section III, the performance of the MP method is reduced when the clutter energy of the selected atom reaches

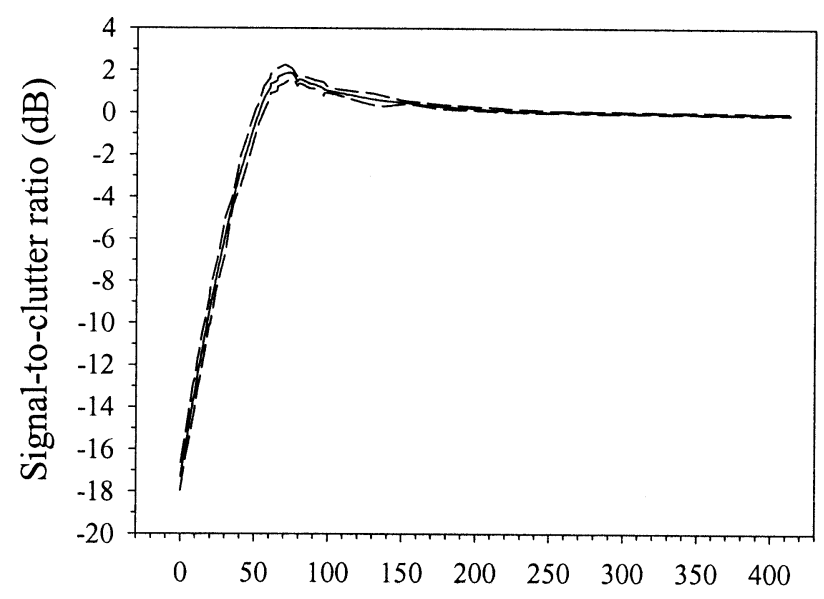

Number of atoms $(L)$ removed from the signal

Fig. 3. Improvement in $S / C$ ratio for low-frequency clutter components following the removal of atoms from the simulated signal $s(n)$. Full line: mean $S / C$ ratio. Dotted lines: mean \pm one standard deviation.

that of the uncorrupted signal $s_{1}(n)$. For the case of transient clutter simulations, the $S / C$ energy ratio was arbitrarily selected to $10.5 \mathrm{~dB}$, and as mentioned earlier, the number of events within a cycle was limited to ten.

\section{Validation of the MP Method With Real Doppler Signals}

The robustness of the MP method was tested with PW Doppler data recorded over the common carotid artery of a normal subject. The cross section of the vessel was visualized with a Sonos 5500 clinical system (Philips Medical Systems, Bothell, WA) at a Doppler angle of approximately $70^{\circ}$. The forward and reverse audio Doppler signals were captured with a 15-6L linear array transducer and digitized at $5 \mathrm{kHz}$ with 12-bit resolution by using a Labview workstation equipped with an analog-to-digital acquisition card (PCI-6024E, National Instruments, Austin, TX). Doppler recordings were performed for at least five cardiac cycles by using the following instrument settings: wall filter $=50 \mathrm{~Hz}$ (minimum value selectable), gate length $=0.05 \mathrm{~cm}$, spectral gain $=75 \%$, power $=0 \mathrm{~dB}$, central spectral baseline, spectral compress $=8$, and spectral reject $=8$. The thermal noise on the sonograms was automatically removed by the thresholding process of the ultrasound instrument. For each recording, the PW sample volume was positioned near the center of the artery.

Recordings with both low-frequency and transient clutter components were digitized for off-line signal processing over five cardiac cycles. Because it was difficult to obtain strong low-frequency clutter vibrations with the instrument because of the use of a wall filter, the following procedure was used to artificially emphasize their impact. The sonogram of each cycle was computed by using the method described earlier (FFTs on 10-ms Hamming windows, lag of $0.5 \mathrm{~ms}$ between each window). The low-frequency clutter components of the amplitude spectra were visually identified and multiplied by 50 (gain of $34 \mathrm{~dB}$ ). Inverse FFTs were then applied to obtain the temporal signals on which the MP algorithm was tested. To obtain transient clutter artifacts, tapping the skin over the neck was performed during recordings. 


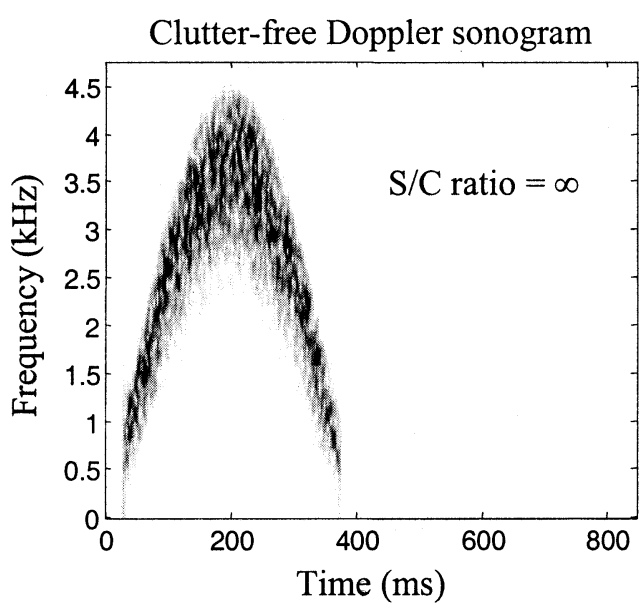

(a)

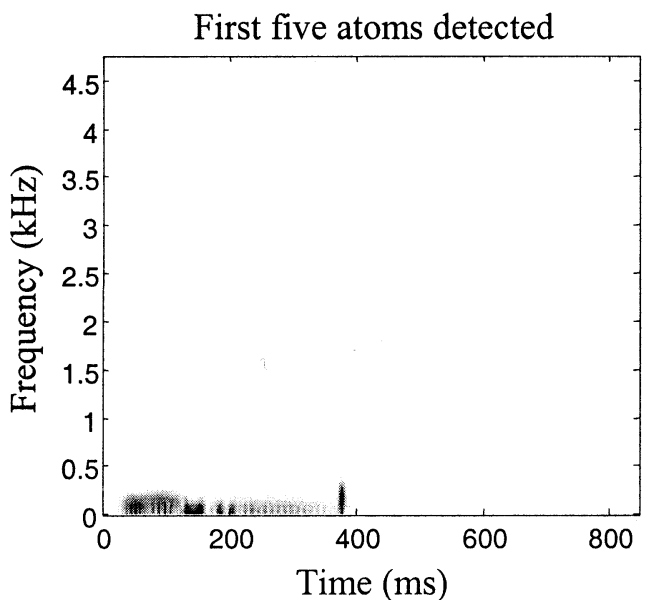

(c)

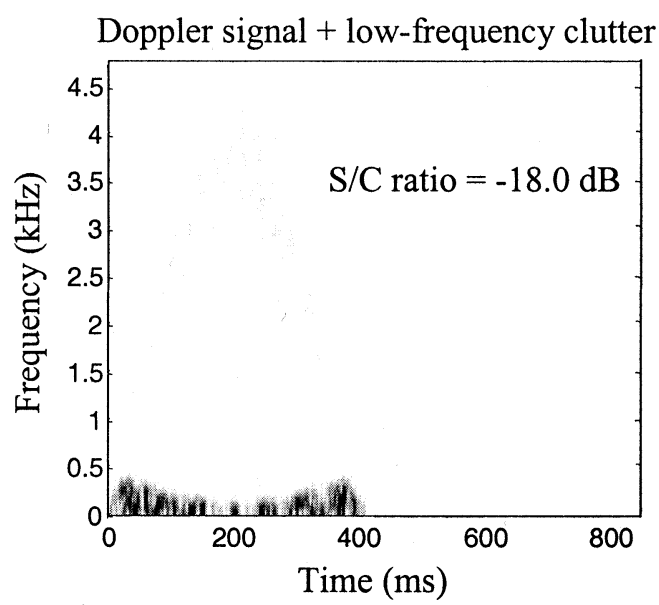

(b)

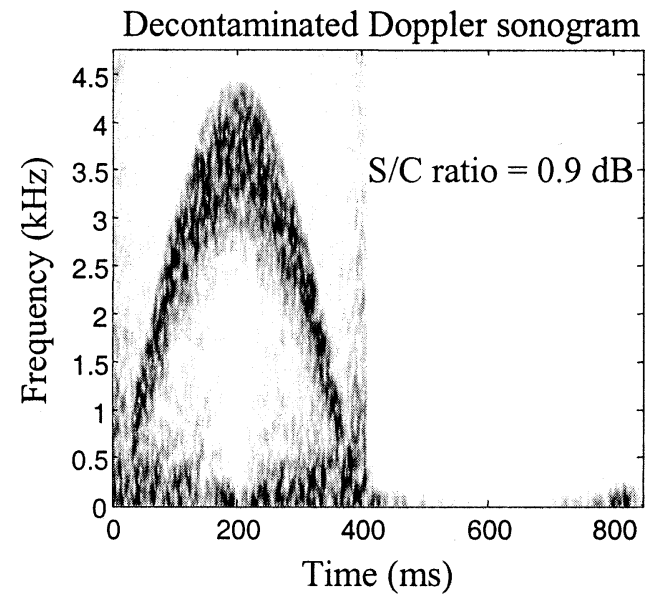

(d)

Fig. 4. Examples of simulated Doppler sonograms for the case of (a) clutter-free flow signal, (b) flow signal contaminated with low-frequency clutter components, (c) the first five atoms that were extracted from the contaminated signal, and (d) the decontaminated signal following the removal of 72 atoms. The grayscales of the sonograms were compressed to a dynamic range of $10 \mathrm{~dB}$ to emphasize the contrasts. No background noise was present in the simulations.

The method described in (11) could not be used to evaluate the performance of the MP method with real data because the signal $s_{1}(n)$ was unknown. Let us consider the energy of the first atom extracted from the signal and define it as $E_{\max }$. An alternative to (11) was to compare the energy of successive atoms $E_{i}$, removed from the Doppler signal, to $E_{\max }$. Thus, the energy ratio $E_{i} \times 100 / E_{\max }$ was computed and the iterative selection of the atoms was stopped when this ratio became smaller than a predetermined threshold of $0.1 \%$.

\section{RESULTS}

\section{A. Performance of the MP Method to Remove Simulated Low-Frequency Clutter Components}

Fig. 3 shows the improvement in $S / C$ ratio as a function of the number of atoms removed from the simulated signal $s(n)$. After removing the first atoms associated to the low-frequency clutter components, the $S / C$ ratio rapidly increased to reach a maximum at $72.0 \pm 4.5$ atoms. Following this maximum, the $S / C$ ratio slightly dropped to reach a plateau following the removal of 250 atoms, approximately. The maximum improvement in $S / C$ ratio was $19.0 \pm 0.6 \mathrm{~dB}$.
Fig. 4 presents Doppler sonograms of (a) the clutter-free Doppler signal $s_{1}(n)$, (b) the signal $s(n)=s_{1}(n)+s_{2}(n)$ contaminated with low-frequency clutter components, (c) the first five atoms that were extracted from $s(n)$, and (d) the decontaminated signal $s_{d}(n)$ following the removal of 72 atoms from $s(n)$. The acceleration and deceleration of the flow are clearly seen from the clutter-free simulated signal. However, as seen on Fig. 4(b), the strong echoes from the low-frequency clutter highly hide the flow components. The efficiency of the MP algorithm can be appreciated in Fig. 4(c). For instance, as expected, the first five atoms were identified among the low-frequency clutter components and not from the flow signal. Although the low-frequency clutter components are highly attenuated from the decontaminated signal, some frequency bins are still visible at low frequencies [Fig. 4(d)]. Nevertheless, the improvement in $S / C$ ratio is significant in this example.

\section{B. Performance of the MP Method to Remove Simulated Transient Clutter Components}

A different strategy was adopted to remove the short transient clutter components from $s(n)$. Since the amplitude of the 


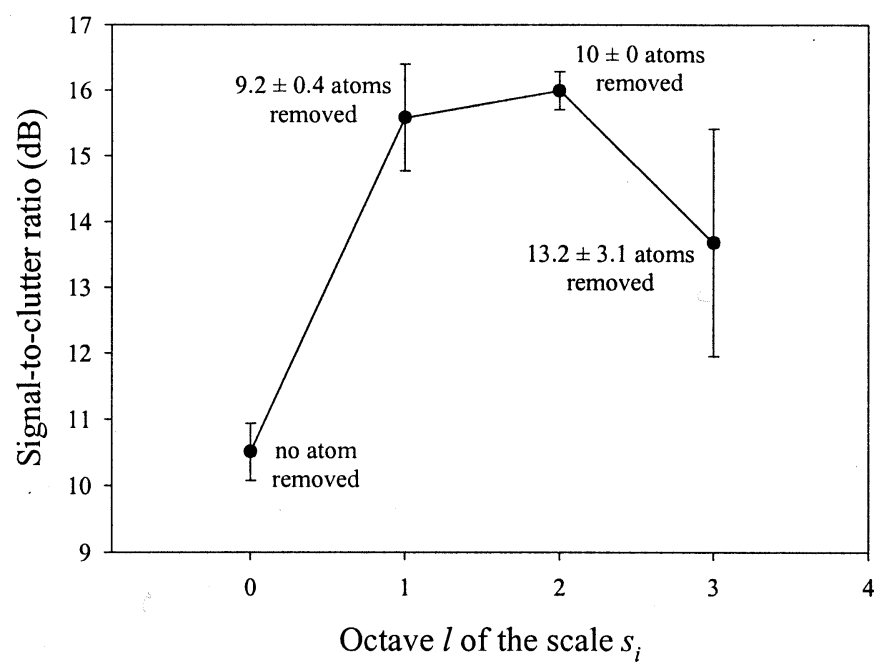

Fig. 5. Improvement in $S / C$ ratio for simulated transient clutter components as a function of the octave of the scale $s_{i}$. Results are expressed in terms of mean \pm one standard deviation.

clutter $\sqrt{E\left(n_{q}\right)}$ in (1c) was set equal to the absolute value of the clutter-free maximum amplitude $\gamma_{m}(n)$, it was inappropriate to associate the clutter components to only one criterion, i.e., the strongest atoms selected. A second criterion was introduced to take into consideration the a priori information known about transient clutters, which is their short duration. Consequently, only the strongest atoms with short durations $[l<3$, where $l$ is the octave of the scale $s_{i}$ in (7)] were subtracted from the signal $s(n)$. Fig. 5 shows the improvement in $S / C$ ratio as a function of the octave of the scale $s_{i}$. After removing $9.2 \pm 0.4$ atoms with $l=1$, the $S / C$ ratio went from $10.5 \pm 0.4 \mathrm{~dB}$ to $15.6 \pm 0.8 \mathrm{~dB}$. Removing an additional $0.8 \pm 0.4$ atoms with $l=2$ increased the $S / C$ ratio to a maximum of $16.0 \pm 0.3 \mathrm{~dB}$. A reduction of the $S / C$ ratio was observed when atoms with $l=1,2$ and 3 were removed from the signal. Thus, the maximum improvement in $S / C$ ratio was $5.5 \pm 0.5 \mathrm{~dB}$, and this was reached by removing exactly ten atoms from $s(n)$, with $l=1$ and $l=2$.

Fig. 6 gives examples of the performance of the MP method to decontaminate the simulated Doppler signal from transient clutter components. Fig. 6(a) shows the clutter-free sonogram on which ten transient clutter Dirac components were superimposed. After removing ten atoms with $l=1$ and $l=2$, the appearance of the sonogram was improved. However, as shown in Fig. 6(b), some transient signals were still present and could not be removed by applying the MP method.

\section{Validation of the MP Method With Real Doppler Data Contaminated With Low-Frequency Clutter}

The energy ratio $E_{i} \times 100 / E_{\max }$ of the atoms removed to decontaminate the real Doppler data from the low-frequency clutter components is shown in Fig. 7. The MP algorithm was applied to both the forward and reverse flow samples. As shown on the figure, the ratio $E_{i} / E_{\max }$ in percent decreased for the first high-energy atoms selected from the Doppler signal. Exponentially decaying relationships were observed. A ratio of $0.1 \%$ was reached, for all five flow cycles studied, following the removal of 15 atoms. This arbitrary threshold $(0.1 \%)$ was

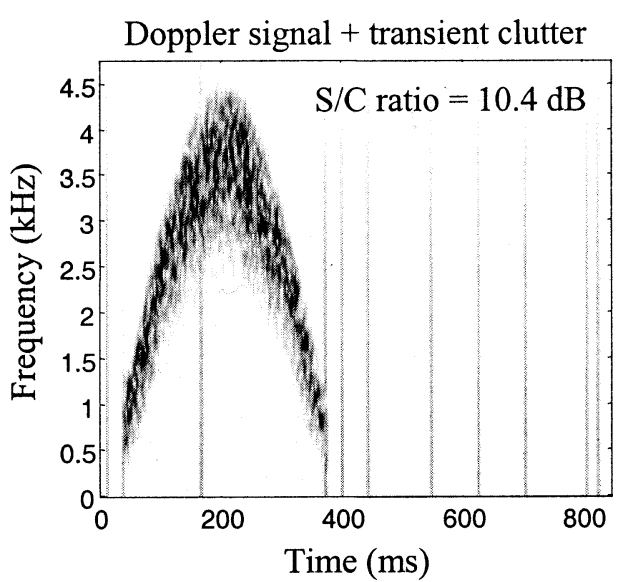

(a)

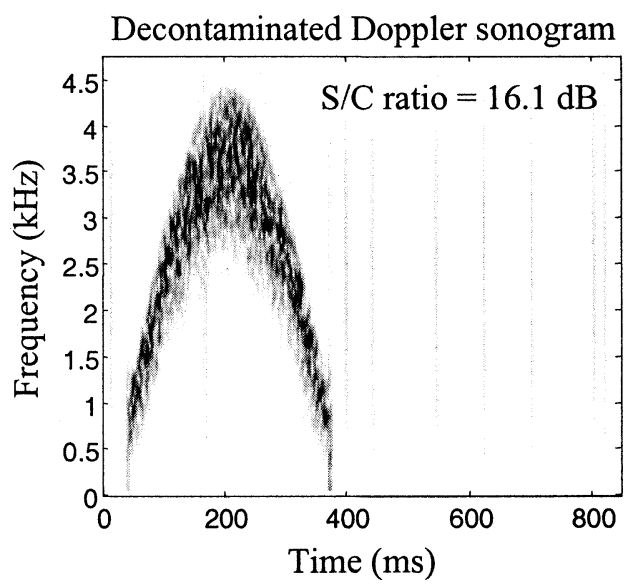

(b)

Fig. 6. Examples of simulated Doppler sonograms of (a) flow signal contaminated with transient clutter components, and (b) the decontaminated signal following the removal of ten atoms. The grayscales of the sonograms were compressed to a dynamic range of $10 \mathrm{~dB}$ to emphasize the contrasts. No background noise was present in the simulations.

selected to decontaminate the Doppler sonogram. As seen in Fig. 8(a), the flow components were not visible from the PW Doppler sonogram contaminated with clutter. This is due to the fact that the low-frequency clutter components, observed close to $100 \mathrm{~ms}$ after the beginning of the cardiac cycle, were artificially amplified by $34 \mathrm{~dB}$ to test the MP algorithm. Removing 15 atoms from both the forward and reverse signals significantly improved the appearance of the sonogram [Fig. 8(b)].

\section{Validation of the MP Method With Real Doppler Data Contaminated With Transient Clutter}

Because the energy of the transient clutter components was close to that of the flow signal, applying the $E_{i} / E_{\max }$ ratio criterion was meaningless. To demonstrate the feasibility of the MP algorithm to remove real transient components, several tries were performed by increasing the number of atoms to remove. Several values of $l$ were also tested. The results shown in Fig. 9 were obtained with 238 atoms with $l<7$. This number of atoms was removed from both the forward and reverse Doppler signals. The performances were similar for the other cardiac cycles recorded. 


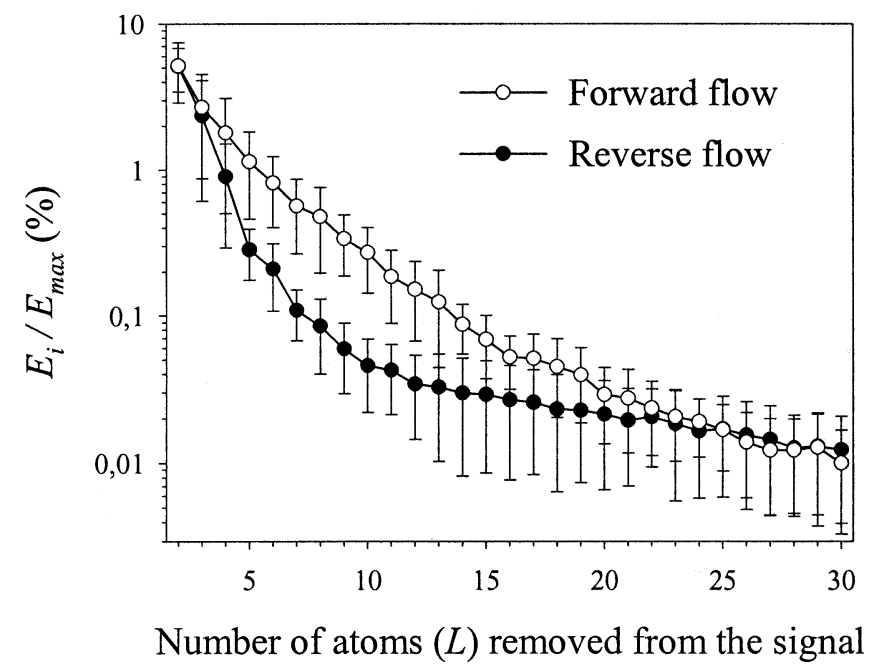

Fig. 7. Changes in the energy ratio $E_{i} / E_{\max }$ of the atoms removed from the Doppler data recorded over the common carotid artery of a normal subject. The removal of low-frequency clutter components with the MP method was applied to both the forward and reverse flow samples. Results are expressed in terms of mean \pm one standard deviation. Note that $E_{i}$ is the energy of successive atoms removed from the PW Doppler signal, and $E_{\max }$ is the energy of the first atom extracted from it. The MP method extracts atoms with a decreasing energy order.

\section{DISCUSSION}

The current study demonstrated the potential benefit of the MP method to remove low-frequency and transient clutter components from PW Doppler signals. This signal processing method may be an alternative to conventional high-pass clutter filters used by ultrasound manufacturers, or to other filtering strategies proposed by research groups [1], [2], [8], [13] (it should be noted that most of the new methods applies to color flow). To our knowledge, the MP algorithm has never been applied for such purpose in the ultrasound literature. Recently, it was used to detect microembolic signals [14], and to characterize turbulent flow with PW Doppler ultrasound [15].

According to Figs. 3 and 5, an optimal number of atoms, or atom duration, must be selected to get the best performance with the MP method. If the number of atoms, or octave $l$, is too small, clutter components remain on the decontaminated signal. On the other hand, if these values are too high, the atoms may be removed from both the clutter and flow signals, which can affect the accuracy of the flow velocity measurements. In Fig. 3, the time-frequency components were removed from either the clutter or flow signal beyond 72 atoms, approximately, since the $S / C$ ratio reached a constant. For the transient clutter components (Fig. 5), selecting the value of $l$ too small did not allow to adequately reduce the transient artifacts, whereas increasing $l$ above three resulted in the removal of atoms mainly from the flow signals.

In the simulation study, it was straightforward to determine the best parameters to select by monitoring the $S / C$ ratio changes. However, to implement this method with real Doppler data, a different strategy had to be implemented. The energy $E_{i}$, of the successive atoms that were removed from the Doppler signal, was compared to $E_{\max }$, which was defined as the energy of the first atom selected. The iterative selection of the atoms to remove low-frequency clutter artifacts was

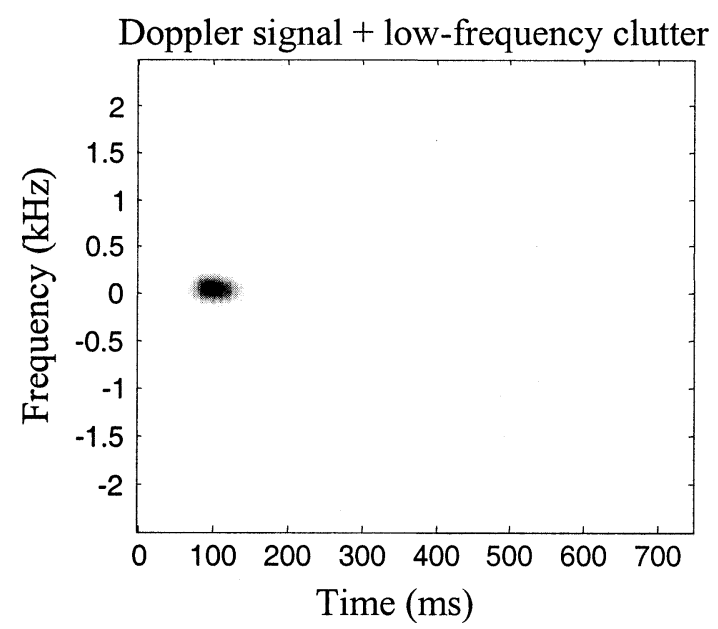

(a)

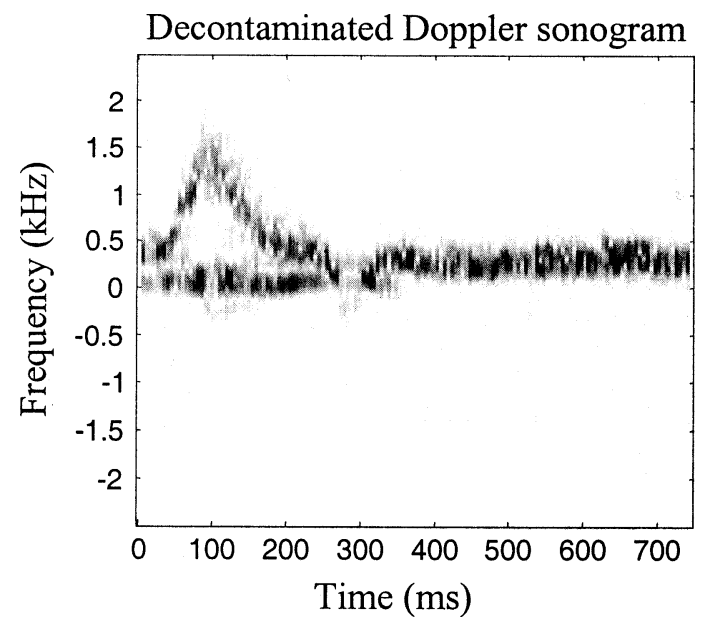

(b)

Fig. 8. Examples of a real Doppler sonogram with (a) low-frequency clutter amplified by a factor of $34 \mathrm{~dB}$, and (b) of the same preamplified sonogram following the removal of 15 atoms. The PW Doppler recordings were performed over the common carotid artery of a normal subject. The grayscales of the sonograms were compressed to a dynamic range of $10 \mathrm{~dB}$ to emphasize the contrasts. Thresholding was performed by the instrument to remove the background noise on the sonograms.

stopped when $E_{i} / E_{\max }$ reached a value of $0.1 \%$. Of course, this threshold of $0.1 \%$ is arbitrary and, as seen in Fig. 8, is qualitatively optimal only for the carotid flow data acquired in the present study. This threshold would have to be validated over a larger database before implementing the method. For transient artifacts superimposed on real Doppler signals, it would be relevant, for real-time implementation on an instrument, to have the possibility (a knob for example) to adjust the octave parameter $l$.

As seen in Figs. 4(d) and 6(b), although the position and frequency bins associated to the clutter are well identified with the MP method, residual errors are visible from the decontaminated sonograms. For the low-frequency clutter simulation, because up to 300 sinusoids were used to generate the signal at a given time instant $n[(1 \mathrm{~b})]$, removing all clutter components was impossible. As shown in Fig. 4(d), some low-frequency signal components are still superimposed over the flow signal. Moreover, random artifacts (similar to background noise) are generated following the removal of time-frequency atoms. If 


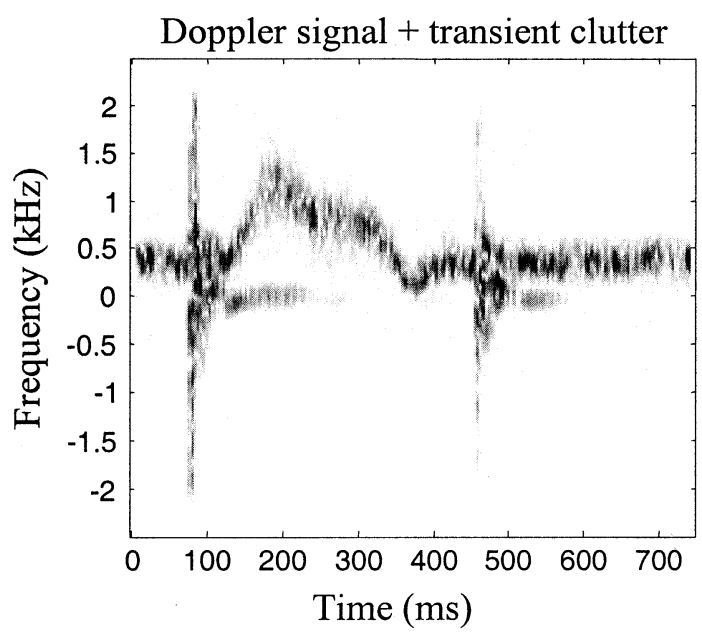

(a)

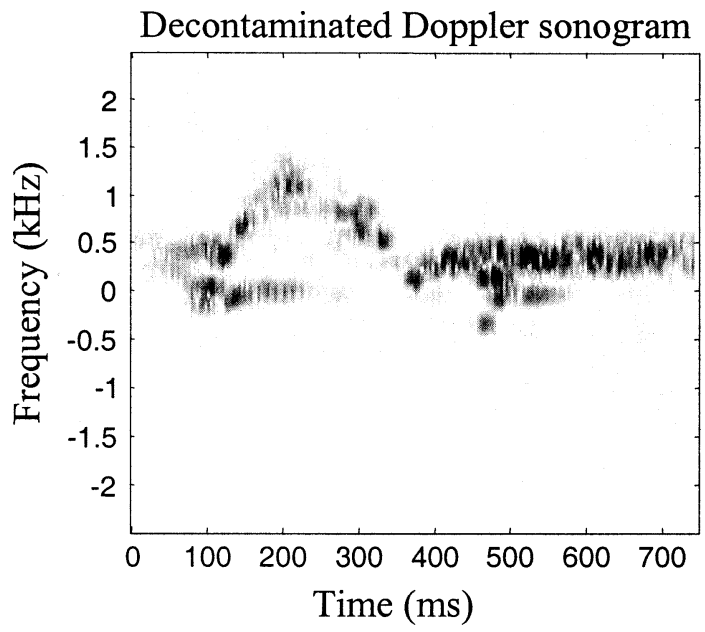

(b)

Fig. 9. Examples of a real Doppler sonogram with (a) transient clutter produced by tapping the skin over the neck, and (b) of the same sonogram following the removal of 238 atoms with $l<7$. The PW Doppler recordings were performed over the common carotid artery of a normal subject. The grayscales of the sonograms were compressed to a dynamic range of $10 \mathrm{~dB}$ to emphasize the contrasts. Thresholding was performed by the instrument to remove the background noise on the sonograms.

the amplitude $\alpha_{i}$, frequency $f_{i}$, and phase $\theta_{i}$ of the selected atoms [(5) and (8)] do not perfectly coincide with the clutter components of the signal $s(n)$, subtracting these atoms from $s(n)$ may effectively generate some level of background noise. A method that can be used to reduce this effect is to apply an amplitude threshold to display the sonogram, as it is performed by most ultrasound instruments. As seen in Figs. 8(b) and 9(b), although better than the simulations, some residual clutter components are still present on the common carotid Doppler sonogram following the use of the MP method. In theory, although the low-frequency flow signal and clutter components are superimposed, the MP method may still be able to remove the clutter while preserving the flow waveform. This should be possible if the frequency and phase of the selected atom do not coincide with those of the flow frequency bins.

An explanation may be given for the fact that the MP method could not perfectly remove transient clutter components from the simulated signal $s(n)$ [Fig. 6(b)]. This may be attributed to the limited time resolution of the MP method to match that of the Dirac impulses used in (1c). The performance of the MP method with simulated signals would have probably been better if the transient clutter components would have lasted more than a fraction of a millisecond, as it was the case here. This expectation was confirmed by applying the MP method to real data [Fig. 9(b)]. Because the transient signals obtained by tapping the skin of the neck above the carotid artery lasted more than a few milliseconds, the performance was better. Very interestingly, the temporal position of each Dirac impulse superimposed over the simulated signal was precisely identified by the MP method. Moreover, ten atoms were optimally extracted from the signal, which correspond exactly to the number of transient components simulated.

In the current study, the feasibility of the MP method to remove clutter was demonstrated with simulated and real PW Doppler data. The number of samples available for signal processing in PW mode is much larger than in color flow. It would thus be of high interest to extend this study to test the feasibility of the MP method for color flow imaging. This should be feasible because of the good time resolution of the MP algorithm. As reported by Torp [2], the phase shift occurring in the transition band of classical high-pass clutter filters can produce bias in the center frequency (color velocity) estimate. This would be another advantage of the MP method because the successive atom removal does not produce phase shifting artifact.

The MP software developed by Mallat and Zhang [11] was run on a Pentium III $(866 \mathrm{MHz})$ computer. This software is written in the $\mathrm{C}$ language and is available on the Web (LastWave package, http://www.cmap.polytechnique.fr). The removal of 100 atoms from the simulated signal $s(n)$ (8142 samples) with this package took around $10 \mathrm{~s}$. New algorithms proposed in the literature [16], faster computers, and computation over less samples should improve the time efficiency of the MP method.

\section{CONCLUSION}

The very few samples of signal available in real-time color-flow imaging and audio Doppler signal analysis pose a difficulty for system designers of clutter filters. This is usually regarded as one of the most difficult technical challenges to overcome, especially for color flow imaging [1]. Another difficulty with conventional high-pass clutter filters is the fact that they are not adaptable to the time-varying characteristics of the clutter and flow signals. The MP method evaluated in the current study, with PW Doppler data, solves some limitations of classical filtering strategies. However, it should be noted that this technique may not allow real-time processing for some time. Nevertheless, this approach should be considered in the future mainly because the signal is decomposed into atoms with decreasing energy order, and because of its good time-frequency resolution that allows transient clutter rejection. Very interestingly, this algorithm can be applied to both the audio Doppler and radio-frequency signals without conceptual modification. It is expected that it may allow clutter removal on color flow signals.

\section{REFERENCES}

[1] D. H. Evans and W. N. McDicken, Doppler Ultrasound: Physics, Instrumentation and Signal Processing, 2nd ed. New York: Wiley, 2000. 
[2] H. Torp, "Clutter rejection filters in color flow imaging: a theoretical approach," IEEE Trans. Ultrason., Ferroelect., Freq. Contr., vol. 44, pp. 417-424, Mar. 1997.

[3] S. Bjaerum, H. Torp, and K. Kristoffersen, "Clutter filter design for ultrasound color flow imaging," IEEE Trans. Ultrason., Ferroelect., Freq. Contr., vol. 49, pp. 204-216, Mar. 2002.

[4] A. P. Oppenheim and R. W. Schafer, Digital Signal Processing. Englewood Cliffs, NJ: Prentice-Hall, 1975.

[5] J. E. Powers, P. N. Burns, and J. Souquet, "8. Imaging instrumentation for ultrasound contrast agents," in Advances in Echo Imaging Using Contrast Enhancement, 2nd ed, N. C. Nanda, R. Schlief, and B. B. Goldberg, Eds. Norwell, MA: Kluwer, 1997, pp. 139-170.

[6] B. Dunmire, K. W. Beach, K. H. Labs, M. Plett, and D. E. Strandness Jr., "Cross-beam vector Doppler ultrasound for angle-independent velocity measurements," Ultrasound Med. Biol., vol. 26, no. 8, pp. 1213-1235, 2000.

[7] A. Heimdal and H. Torp, "Ultrasound Doppler measurements of low velocity blood flow: Limitations due to clutter signals from vibrating muscles," IEEE Trans. Ultrason., Ferroelect., Freq. Contr., vol. 44, pp. 873-881, July 1997.

[8] D. B. Keenan, F. J. Owens, M. S. Murphy, and D. Need, "Removal of wall-motion artefacts in Doppler ultrasound signals using linear prediction filtering," Electron. Lett., vol. 35, pp. 347-349, Feb. 1999.

[9] L. Y. L. Mo and R. S. C. Cobbold, "A nonstationary signal simulation model for continuous wave and pulsed Doppler ultrasound," IEEE Trans. Ultrason., Ferroelect., Freq. Contr., vol. 36, pp. 522-530, Sept. 1989.
[10] Z. Guo, L. G. Durand, and H. C. Lee, "Comparison of time-frequency distribution techniques for analysis of simulated Doppler ultrasound signals of the femoral artery," IEEE Trans. Biomed. Eng., vol. 41, pp. 332-342, Apr. 1994.

[11] S. G. Mallat and Z. Zhang, "Matching pursuits with time-frequency dictionaries," IEEE Trans. Signal Processing, vol. 41, pp. 3397-3415, Dec. 1993.

[12] P. D. Welch, "The use of fast Fourier transform for the estimation of power spectra: A method based on time averaging over short, modified periodograms," IEEE Trans. Audio Electroacoustic, vol. AE-15, no. 6, pp. 70-73, 1967.

[13] L. A. F. Ledoux, P. J. Brands, and A. P. G. Hoeks, "Reduction of the clutter component in Doppler ultrasound signals based on singular value decomposition: A simulation study," Ultrason. Imag., vol. 19, pp. 1-18, 1997.

[14] G. Devuyst, J. M. Vesin, P. A. Despland, and J. Bogousslavsky, "The matching pursuit: A new method of characterizing microembolic signals," Ultrasound Med. Biol., vol. 26, no. 6, pp. 1051-1056, 2000.

[15] G. Cloutier, D. Chen, and L. G. Durand, "Performance of time-frequency representation techniques to measure blood flow turbulence with pulsed-wave Doppler ultrasound," Ultrasound Med. Biol., vol. 27, no. 4, pp. 535-550, 2001.

[16] R. Gribonval, "Fast matching pursuit with a multiscale dictionary of Gaussian chirps," IEEE Trans. Signal Processing, vol. 49, pp. 994-1001, May 2001. 